\title{
Tomato Plants Overexpressing a Celery Mannitol Dehydrogenase (MTD) Have Decreased Susceptibility to Botrytis cinerea
}

\author{
Takshay K. Patel', Sergei F. Krasnyanski'1, George C. Allen'1, Frank J. Louws², \\ Dilip R. Panthee ${ }^{1}$, John D. Williamson ${ }^{1 *}$ \\ ${ }^{1}$ Department of Horticultural Science, North Carolina State University, Raleigh, NC, USA \\ ${ }^{2}$ Department of Plant Pathology, North Carolina State University, Raleigh, NC, USA \\ Email: ${ }^{*}$ john williamson@ncsu.edu
}

Received 30 March 2015; accepted 9 May 2015; published 13 May 2015

Copyright (C) 2015 by authors and Scientific Research Publishing Inc.

This work is licensed under the Creative Commons Attribution International License (CC BY). http://creativecommons.org/licenses/by/4.0/

(c) (i) Open Access

\section{Abstract}

The oxidative burst is a critical early event in plant-pathogen interactions that leads to a localized, programmed cell death (PCD) called the hypersensitive response (HR). The HR and associated PCD retard infection by biotrophic pathogens, but can, in fact, enhance infection by necrotrophic pathogens like Botrytis cinerea. In addition to signaling the induction of the HR, reactive oxygen species (ROS) produced during the oxidative burst are antimicrobial. We hypothesize that pathogens such as $B$. cinerea survive the antimicrobial effects of ROS, at least partially by secreting the antioxidant mannitol during infection. This is supported by the previous observation that overexpression of the catabolic enzyme mannitol dehydrogenase (MTD) can decrease a plants susceptibility to mannitol-secreting pathogens like $B$. cinerea. To extend the above hypothesis, and test the general utility of this approach in an important horticultural crop, we overexpressed celery MTD in tomato (Solanum lycopersicum cv. "Moneymaker"). In these studies, we observed a significant increase (up to $\mathbf{9 0 \%}$ ) in resistance to $B$. cinerea in transgenic tomatoes expressing high amounts of MTD.

\section{Keywords}

Hypersensitive Response, Pathogenesis Response, Plant-Pathogen Interaction, Programmed Cell Death, Reactive Oxygen Species

\footnotetext{
${ }^{*}$ Corresponding author.
}

How to cite this paper: Patel, T.K., Krasnyanski, S.F., Allen, G.C., Louws, F.J., Panthee, D.R. and Williamson, J.D. (2015) Tomato Plants Overexpressing a Celery Mannitol Dehydrogenase (MTD) Have Decreased Susceptibility to Botrytis cinerea. American Journal of Plant Sciences, 6, 1116-1125. http://dx.doi.org/10.4236/ajps.2015.68116 


\section{Introduction}

Botrytis cinerea is a necrotrophic fungus with a documented host range of more than 200 plant species [1] [2]. This broad host range is likely due, at least in part, to the wide range of virulence factors that have been described for B. cinerea, including enzymes and toxins that aid in penetrating and killing host cells [2]. Normally, the hypersensitive response (HR), a localized, programmed cell death (PCD), is one of the plants primary defenses against pathogen attack. The HR is initiated by the rapid, post-infection production of reactive oxygen species (ROS) and subsequent induction of PCD that limits the spread of infection [3] [4]. Interestingly, while it is an important defense against biotrophic pathogens, HR facilitates infection by necrotrophic pathogens like $B$. cinerea [5]. This is supported by the observation that HR-deficient Arabidopsis mutants are less susceptible to $B$. cinerea [6]. Further, B. cinerea is itself reported to have an NADPH oxidase and a superoxide dismutase (SOD) [7] [8] that are activated, and thus presumably produce superoxide and hydrogen peroxide, respectively, as a part of the infection process. We hypothesize that this additional ROS produced by $B$. cinerea can amplify the host plant HR/PCD and thus increase physical access for infection. Although induction of HR by the oxidative burst provides $B$. cinerea access to the plant, ROS are also very effective antimicrobial/antifungal agents [4]. To successfully infect a plant, $B$. cinerea must, therefore, survive not only the effects of ROS produced as a part of the plant's oxidative burst, but also its own ROS. This implies that $B$. cinerea has very effective protective mechanisms against ROS.

Previous work found that B. cinerea, as well as other fungal pathogens such as Alternaria alternata, Uromyces fabae and Cladosporium fulvum [9]-[12] secrete mannitol during the infection process, presumably to buffer the effects of ROS. This is based on the hypothesis that mannitol is, amongst other things, an antioxidant. Smirnoff and Cumbes [13], for instance, initially reported that mannitol quenched hydroxyl radicals $(\cdot \mathrm{OH})$ generated in an in vitro assay. In vivo, Saccharomyces cerevisiae mutants that lacked endogenous ROS quenchers but produced mannitol via expression of a bacterial $m t l D$ were able to grow on media with high concentrations of $\mathrm{H}_{2} \mathrm{O}_{2}$ [14]. The role of mannitol as a necessary pathogenicity factor is further supported by a number of in vivo plant studies. For instance, Joosten et al. [12] observed that one difference between virulent and avirulent strains of $C$. fulvum was the presence of mannitol in the apoplast of tomato leaves during infection by the virulent strain. More direct evidence from Vélëzet al. [15] showed that mutant strains of A. alternata deficient in mannitol production became avirulent. Finally, mannitol secretion by both $B$. cinerea and A. alternata is induced in the presence of plant extracts [9] [10], suggesting that mannitol is an important extracellular pathogenicity factor for these fungi.

In response to infection by mannitol-secreting fungal pathogens, a number of plants have been found to secrete the mannitol catabolic enzyme, mannitol dehydrogenase (MTD). In the plant kingdom there are over 100 species that use mannitol as a carbon and energy source [16]. In these mannitol-utilizing plants, the catabolic enzyme MTD, a 1-oxidoreductase, oxidizes mannitol to mannose for entry into central metabolism [17]. Mtd homologs surprisingly are also found in plants that do not make or utilize mannitol, such as Arabidopsis, tobacco, and tomato [18]-[20], although expression of these homologs is typically low. Increased MTD expression is also triggered in these plants, as well as in celery, by inducers of the pathogenesis response (PR gene inducers) such as salicylic acid (SA), INA (2,6-dichloroisonicotinic acid) and fungal elicitors [19]. This implies that, in addition to its metabolic role in plants like celery and parsley [17], Mtd is also a PR-gene in both mannitol and non-mannitol plants [18] [19] [21]. Interestingly, SA not only induces MTD expression; it also induces the secretion of MTD into the extracellular space or apoplast [22] [23]. This presumably facilitates co-localization of the normally cytosolic/symplastic MTD with the apoplastically localized mannitol secreted by fungal pathogens.

Our current hypothesis is that the survival of $B$. cinerea during the oxidative burst, and thus its ability to infect, is at least partly due to its ability to secrete mannitol and thus protect itself against the effects of antimicrobial ROS. Removal of this protective mannitol should, therefore, allow destruction of the invading fungus early during the oxidative burst. So, if plants produce and secrete MTD to counter infection by mannitol-secreting fungi, why are plants like tomato still susceptible to $A$. solani and B. cinerea, even though they have pathogen-induced Mtd homologs? Given that MTD overexpression protects tobacco and zonal geranium against A. alternata and $B$. cinerea, respectively [9] [10], and that native MTD expression in plants that do not produce mannitol can be quite low [19], it is thought that the limited MTD present in these plants is easily saturated by high levels of fungal mannitol secretion.

Taken together, this suggests that, while MTD induction and secretion could be an important resistance me- 
chanism against mannitol-secreting fungi, relatively high amounts of MTD might be required. This further implies that overexpression of MTD could be an effective means of providing broad resistance against mannitolsecreting necrotrophic fungi. In the following study we test and extend the hypothesis that overexpressing MTD in tomato (Solanum lycopersicumcv. "Moneymaker") increases resistance to mannitol-secreting fungal pathogens by evaluating potential changes in resistance to the mannitol-secreting pathogen $B$. cinerea in first and second generation transgenic plants overexpressing MTD.

\section{Materials and Methods}

\subsection{Tomato Transformation}

Tomato seeds (Solanum lycopersicum cv. "Moneymaker”; Thompson and Morgan, Aurora, IN) were surface sterilized with 70\% ethanol for $1 \mathrm{~min}$, then with 50\% Clorox (commercial bleach) for 30 min and finally rinsed 3 times with sterile distilled water. Sterilized seeds were allowed to germinate in Magenta ${ }^{\mathrm{TM}}$ GA-7 boxes on agar (12 g/L) (Caisson Laboratories Inc., North Logan, UT) medium containing MS salts [24] with Gamborg's vitamins [25] and sucrose $(10 \mathrm{~g} / \mathrm{L})$ at $\mathrm{pH}$ 5.6. The seeds were germinated at $26^{\circ} \mathrm{C}$ with a $16 / 8 \mathrm{hr}$ light/dark cycle. Cotyledons and hypocotyls of 7-day old seedlings were used as a source of explants for transformation.

A $500 \mu \mathrm{l}$ aliquot of a frozen stock of Agrobacterium tumefaciens strain GV3101 harboring the plasmid construct pTN214N8 [9] was inoculated into 25 ml liquid Yeast Extract Peptone (YEP) with 50 mg/L gentamycin, $100 \mathrm{mg} / \mathrm{L}$ spectinomycin and $10 \mathrm{mg} / \mathrm{L}$ rifampicin and grown overnight on a shaker at $220 \mathrm{rpm}$ at $28^{\circ} \mathrm{C}$. This overnight culture was centrifuged and the resulting bacterial pellet resuspended in $25 \mathrm{ml}$ liquid suspension (RL) medium containing MS salts with Gamborg's vitamins, sucrose (30 g/L), glucose (2 g/L), and 40 mg/L acetosyringone at $\mathrm{pH} 5.6$, and grown for another $1-2 \mathrm{hr}$ at $28^{\circ} \mathrm{C}$. For explant inoculation, this culture was diluted to achieve a cell density that gave an $\mathrm{OD}_{600}$ of $\sim 0.3$ (ca. $3 \times 10^{7}$ cells $/ \mathrm{ml}$ ).

Seedling explants with hypocotyls and well-shaped (not curly) cotyledons were collected in a sterile Petri dish containing a small amount of RL medium. Explants were then placed on a solid co-cultivation medium (TCC) [MS salts, Gamborg's vitamins, sucrose (30 g/L), glucose (2 g/L), 1-naphthaleneacetic acid (NAA) (0.1 mg/L), benzyladenine (BA) (1 mg/L), 2-(N-morpholino) ethanesulfonic acid (MES) $(700 \mathrm{mg} / \mathrm{L})$, acetosyringone (40 $\mathrm{mg} / \mathrm{L})$ and agar $(10 \mathrm{~g} / \mathrm{L})$ at $\mathrm{pH} 5.6]$ where cotyledons were wounded several times with a needle and hypocotyls cut into 3 - $5 \mathrm{~mm}$ segments. Both types of explants (cotyledons and hypocotyls) were then submerged in $40 \mathrm{ml}$ of the Agrobacteriun suspension described above, and shaken for $15 \mathrm{~min}$ at $50 \mathrm{rpm}$ at room temperature. Following inoculation, cotyledon and hypocotyl pieces were blotted dry on sterile paper towels and placed on TCC medium for $48 \mathrm{hr}$ in the dark at $25^{\circ} \mathrm{C}$.

Following 48 hr co-cultivation, cotyledon and hypocotyl explants were washed 3 times with liquid RL medium containing $300 \mathrm{mg} / \mathrm{L}$ timentin, blotted dry on sterile paper towels and placed on shoot regeneration (TSR) medium [MS salts, Gamborg's vitamins, sucrose (30g/L), indole-3-acetic acid (IAA) (0.1 mg/L), zeatin (2 $\mathrm{mg} / \mathrm{L})$, timentin (300 mg/L), kanamycin (100 mg/L), acetosyringone (40 mg/L), agar (6 g/L) at pH 5.6]. After one week all explants were re-positioned to assure full contact with the medium. Three weeks later all explants were transferred to fresh TSR medium for continued incubation under 20 watt cool-white fluorescent lights with a 16/8 hr light/dark photoperiod.

As soon as shoots had grown to $1-2 \mathrm{~cm}$ they were excised and placed on rootingmedium (TR) containing MS salts, Gamborg's vitamins, sucrose (30 g/L), timentin (300 mg/L), and kanamycin (100 mg/L) for secondary selection. Plants with well-developed roots were transferred into small plastic pots with soil and covered with clear plastic covers for a week in a chamber at $25^{\circ} \mathrm{C}$ under 20 watt cool-white fluorescent lights with a 16/8 hr light/dark photoperiod for acclimatization. After acclimatization plants are transferred to the greenhouse for further growth, fruiting and seed collection.

\subsection{Verifying the Presence of the $M t d$ Transgene}

Kanamycin-resistant shoots were screened for the presence of the 35S-Mtd transgene using PCR primers that amplified a $1.88 \mathrm{~kb}$ fragment extending from inside the 35S promoter to the interior of the nos terminator of the Mtd transgene as previously described [9]. Primers were: 35S-FWD1 and 35S-FWD2; (gaactcgccgtaaagactgg and aaacctcctcggattccatt, respectively), and nosT-REV1 and nosT-REV2 (agggaagaaagcgaaaggag and ttgcgcgctatatttgtt, respectively). A total of forty-four kanamycin-resistant shoots were screened, of which thirty-five 
primary transformants were found to have the Mtd transgene. Nine [9] plants lacked the 35S-Mtd transgene although they were $\mathrm{Km}^{\mathrm{R}}$ (i.e. presumed vector transformants).

\subsection{Protein Extraction and MTD Blot Analyses}

For analysis of $M t d$ transgene expression, leaf tissue from plants [transformed, untransformed wild-type (WT) and vector transformed (VT) controls] was harvested and ground in Bio-Rad (Richmond, CA) SDS sample buffer (1:2 w/v) and protein concentrations determined by the method of Bradford [26]. Proteins were separated by SDS-PAGE [27], blotted onto nitrocellulose, and probed with an anti-MTD serum (1:2000) [28]. Serum crossreacting proteins were visualized using an alkaline phosphatase (AP)-linked secondary antibody (1:2400) (Promega Corp., Madison, WI). Of the plants containing the Mtd transgene that were assessed, 8 expressed high levels of MTD protein, while the rest expressed intermediate to low amounts, although higher than the WT controls.

\subsection{Fungal Growth, Maintenance and Spore Preparation}

Mycelial cultures of the fungal pathogen Botrytis cinerea (a field isolate from the lab of Dr. Michael Benson, Department of Plant Pathology, North Carolina State University) were maintained at room temperature on Potato Dextrose Agar (PDA) (Benton, Dickinson Co., Sparks, MD). A plug from the edge of a spreading culture was transferred to fresh media every 2 weeks for maintenance. To initiate sporulation, a mycelial plug or spore stock was transferred to the center of an oatmeal agar (Benton, Dickinson Co., Sparks, MD) plate and maintained at $21^{\circ} \mathrm{C}$ under an approximately 8/16 hr light/dark photoperiod in a humidity chamber made from a lidded plastic box with wet paper towels lining the bottom. Significant sporulation was present by 10 to 14 days. Sporulating cultures were overlaid with $10 \mathrm{~mL}$ sterile $0.01 \%$ Tween 80 , rubbed with a sterile, bent glass rod to loosen spores and the resulting spore suspensions collected. Spore suspensions were filtered through two layers of sterile cheesecloth, quantified using a hemocytometer and diluted to the desired concentration. For long-term storage spores were stored at $-20^{\circ} \mathrm{C}$ in $15 \%$ glycerol, $0.01 \%$ Tween 80 .

\subsection{Detached Leaf Assay}

A detached leaf assay similar to that described by Spurr [29] was used to assess resistance to B. cinerea. Both young $(2-3 \mathrm{~cm})$ and mature $(6-7 \mathrm{~cm})$ leaves from each transformed genotype were harvested and placed abaxial side up on wire mesh suspended above water in a transparent plastic container with a lid. Five $\mu$ l of $B$. cinerea frozen stock spore suspension (containing $4 \times 10^{6}$ spores $\cdot \mathrm{mL}^{-1}$ ) were spotted onto each leaf at 2 to 6 locations (depending on leaf size) and allowed to dry briefly. These spots were then pierced with a sterile needle to initiate infection. In addition to leaves from Mtd-transformed plants, leaves from WT plants were included in each experimental repetition (i.e. in each box) as a susceptible control. Boxes were then closed and placed inside another plastic box at room temperature with an approximately $16 / 8 \mathrm{hr}$ light/dark photoperiod. Two to six treatments (inoculations) per leaf with 3 experimental units per treatment were replicated at least three times. Symptom development was assessed as described by Floryszak-Wieczorek et al. [30], with the area of each lesion being recorded at $24 \mathrm{hr}$ intervals for 5 days or until lesions merged. Lesion areas were scored using the area function of the Adobe Photoshop ${ }^{\mathrm{TM}}$ software suite. Lesion areas normalized against those of wild type controls in each box were used for comparison of resistance.

\subsection{Seedling Assay}

Seeds from one high and one medium MTD expresser, as well as from VT and WT plants were selected for the seedling assay. Seed from selfed, primary transformants $\left(\mathrm{T}_{0}\right.$ 's) were grown on medium containing $1 / 2 \mathrm{MS}$ salts, kanamycin $(100 \mathrm{mg} / \mathrm{L})$, agar $(6 \mathrm{~g} / \mathrm{L})$ at $\mathrm{pH} 5.6$ to produce $\mathrm{T}_{1}$ generation tomato seedlings/plants. Because this population was still segregating for the Mtd transgene, only plants/seedlings with the Mtd transgene grew on kanamycin selection. WT and VT seeds were grown on similar media without kanamycin. Seedlings $(6-8 \mathrm{~cm})$ were transferred to small plastic pots with soil and covered with clear plastic covers at $25^{\circ} \mathrm{C}$ for a week to acclimate. After acclimation, 12 to $15 \mathrm{~cm}$ seedlings were arranged in a random complete block design consisting of a minimum of six plants per experimental unit for 8 replications and sprayed with $B$. cinerea spores $\left(10^{7}\right.$ spores $\cdot \mathrm{mL}^{-1}$ ). This spore concentration was selected in preliminary experiments by inoculating wild type seedlings with a range of spore concentrations $\left(10^{3}-10^{8}\right.$ spores $\left.\cdot \mathrm{mL}^{-1}\right)$ and selecting the lowest concentration that 
produced robust symptoms on susceptible WT controls. Symptom development was assessed as the percentage of infected leaf tissue area, with the total seedling leaf area being $100 \%$. Infected area was recorded every $24 \mathrm{hr}$ through day 8 post inoculation, or until seedlings started to show new uninfected growth. Differences in resistance were compared using repeated measure analysis and ANOVA in Statistical Analysis Software (SAS, version 9.3, Cary, NC).

\section{Results}

\subsection{Leaves of Transgenic Tomatoes Expressing High Amounts of MTD Are More Resistant to $B$, cinerea}

The amounts of MTD expressed in plants transformed with a MTD-encoding transgene, were determined using protein blot analyses, and compared to levels found in wild type/untransformed (WT), and vector transformed (VT) plants. Transgenic tomato plants selected for further analysis expressed amounts of MTD ranging from high to low, with the lowest levels being comparable to levels in WT plants. If MTD protects against fungi such as $B$. cinerea that secrete mannitol as a pathogenicity factor, higher levels of MTD in transformed plants should provide higher levels of resistance compared to WT. To test this hypothesis, detached leaf assays were used to assess potential differences in symptom development between WT and transgenic plants expressing different amounts of MTD. Susceptible reactions were observed on both WT and low MTD expressing leaves as expanding necrotic lesions around the site of inoculation. In contrast, resistant reactions ranged from little or no visible necrosis to a decreased rate of lesion development when compared to susceptible reactions (examples seen in Figure 1(a)). Lesion development was assessed every $24 \mathrm{hr}$ until day 5 post-inoculation, with lesion area deter-

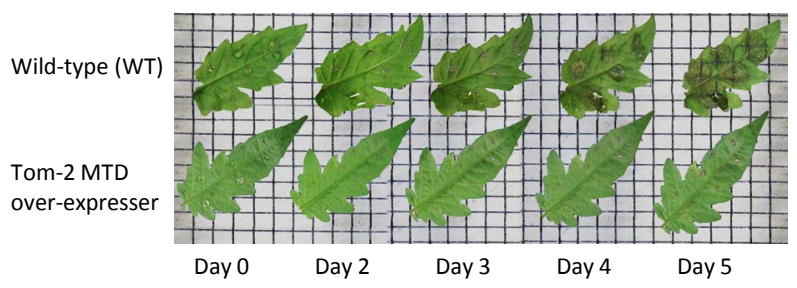

(a)

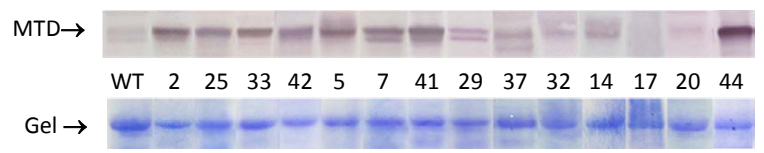

(b)

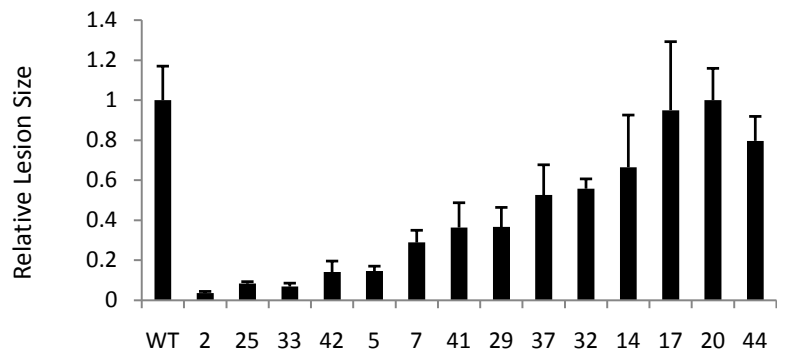

(c)

Figure 1. Assessing B. cinerea resistance using detached leaves from MTD over-expressing tomato. a) Example of lesion development on leaves of an untransformed, wild-type (WT), susceptible plant compared to a transformed, MTD overexpressing plant (Tom-2); (b) Blot analysis of MTD protein in Mtd-transformed and untransformed (WT) controls. Lanes are reordered to correspond to observed levels of resistance in the detached leaf assay (high to low resistance). Coomassie stained lanes are shown to document relative protein loading (Gel), and allow comparison of MTD expression and relative loading; (c) Leaves from Mtd-transformed (2, 25, 33, etc.) and untransformed (WT) plants were inoculated at 5 to 6 spots with $B$. cinerea spores. Infection was scored as lesion area on day 5 post-inoculation for each inoculation site, and normalized against lesion size on untransformed (WT) leaves. Data shown are means \pm SE from at least three independent replications for each transformed plant $(2,25,33$ etc.). 
mined as described above.

Leaves from transgenic plants expressing high amounts of MTD had significantly reduced lesion development compared to leaves from WT plants (plants 2, 25, 33, 42 etc.; Figure 1(b) and Figure 1(c)). Plants expressing intermediate levels of MTD (e.g. plants 29, 14) also showed reduced lesion size, although not as much as the high MTD expressers (e.g. plants 2, 33). Finally, plants like 17 and 20 with MTD levels similar to those of WT plants, showed lesion development similar to WT plants. Although higher levels of MTD largely correlated with decreased lesion size (increased resistance), plants 44 and 41 were exceptions. In particular, plant 44, which expressed extremely high levels of MTD, was no more resistant than WT. Possible reasons for this are addressed in the discussion.

Resistance assays conducted on fully expanded/ mature leaves $(6$ to $7 \mathrm{~cm}$ ) are shown in Figure 1(a). When the same assay was used to test immature leaves $(2$ to $3 \mathrm{~cm}$ ) a similar gain in resistance was seen; although, when normalized against WT, the difference was not as great as that seen for mature leaves. Younger tissues are often reported to be more resistant to pathogens [31] and higher resistance to B. cinerea was, indeed, observed in younger leaves both from transgenic plants and non-transgenic controls. Nonetheless, resistance gain in young and mature leaves showed a strong positive correlation $(\mathrm{R}=0.92)$ (Figure 2$)$.

\subsection{Second Generation Transgenic Seedlings Retain Resistance to $B$, cinerea}

Seedlings $\left(T_{1}\right)$ grown from seed of selfed, primary transformants $\left(T_{0}\right)$ were also tested for resistance to $B$. cinerea, both to assess the effect of MTD overexpression in a whole-plant assay, and to see whether MTD resistance was heritable. Seeds from a selfed high MTD expresser (plant 25), a selfed medium MTD expresser (plant 14), and vector transformed (VT) and WT controls were selected for these assays. As seen in Figure 3, whole plant infection rates for both medium and high expressing plants were lower than for either WT or VT, with reduction in symptoms being particularly significant for seedlings in the high expressing group (group 25; Figure 3). WT and VT seedlings were both ca. $40 \%$ infected by day 8 , although at this point infection had not yet reached a maximum. In contrast, high expressing group 25 plants had reached a maximum infection of $10 \%-15 \%$ by day 4 , and infection did not increase thereafter. After day 4, new uninfected growth was observed on seedlings from group 25, but not on other plants in the test.

\section{Discussion}

Although MTD overexpression in tobacco and zonal geranium [9] [10] has been previously reported to provide resistance to A. alternata and Botrytis cinerea respectively, work presented here is the first report of successful engineering of significant resistance to these necrotrophic pathogens in greenhouse tomato. In addition, results of second-generation seedlings assays (Figure 3 ) indicate that, not only is this resistance stable and heritable, but

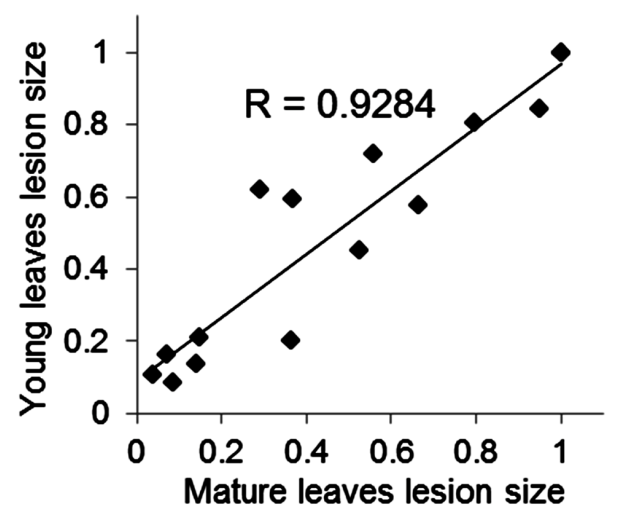

Figure 2. Correlation between resistance in young and mature leaves. Correlation between the lesion sizes for young and mature leaf data was analyzed by simple linear regression. Although not individually labeled, each point on the graph represents lesion size for young and mature leaves for a specific plant (e.g. plant 2, 33 and etc.). As young leaves were innately more resistant, the "relative" resistance of the young transgenic leaves was less than mature leaves because data was normalized against a more resistant "susceptible” control. Even so, a correlation coefficient (R) of 0.9284 indicates a strong positive correlation between resistance of young and mature leaves. 


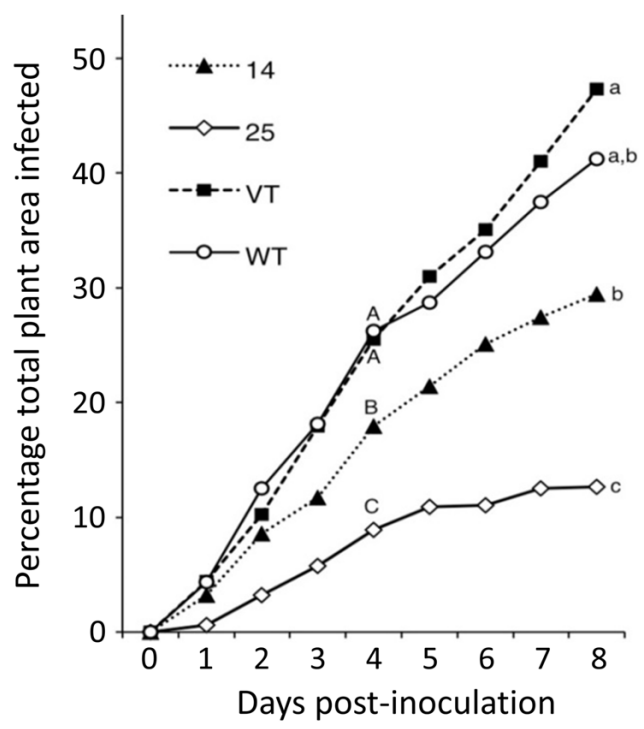

Figure 3. Resistance to $B$. cinerea in second-generation MTD-over-expressing transgenic tomato plants. Seedlings from one high (25) and one medium (14) MTD expresser, vector transformed (VT) and untransformed (WT) tomato plants were assayed. Seedlings were arranged, inoculated and assessed as described in Experimental Procedures. Disease development was scored over a period of 8 days as a relative percentage of the total leaf tissue area, with total plant leaf area being 100 percent. Means for each seedling group are shown. Differences in resistance among the four groups for each day were compared by ANOVA. Means with different letters are significantly different from each other on day 4 and day 8 at $p<0.05$.

that whole plant resistance correlates well with results of detached leaf assays.

Secretion of mannitol during infection is important for full virulence in fungi such as A. alternata and B. cinerea. In addition to previous transgenic studies from our lab [9] [10], a study by Dulermo et al. [32] provides compelling evidence of mannitol's role in B. cinerea pathogenicity. In that study it was observed that, unlike the dramatic loss of virulence reported by Vélëz et al. [15] for mannitol double knockout mutants of A. alternata, a mutant of $B$. cinerea disrupted for the same two mannitol metabolic enzymes still produced mannitol, and was still virulent. This observation led to the discovery of a third mannitol metabolic gene in $B$. cinerea that had only $11 \%$ amino acid homology to the previously described mannitol dehydrogenase (Bcmtdh), but was $84 \%$ homologous to a Tuber borchii (truffle) mannitol dehydrogenase (Tbmtdh) [33]. The presence of this third mannitol biosynthetic gene homolog combined with the continued synthesis of mannitol in the double mannitol biosynthetic mutant support the hypothesis that mannitol is important for $B$. cinerea virulence.

Although there is presently no data on the virulence of $B$. cinerea triple knockout mutants, the gain in resistance of MTD overexpressing plants observed here supports a role for mannitol in the $B$. cinerea infection process. The exact mechanism whereby mannitol functions as a virulence factor is, however, still a matter of debate. We had previously hypothesized that mannitol, being an efficient antioxidant, quenched ROS that signaled induction of global plant defenses. Unfortunately, this model is not consistent with a number of recent observations concerning the role of ROS and the induction of HR during B. cinerea infection [2] [5] [7] [8]. These studies indicate that, because ROS and the associated induction of the HR actually enhance infection by $B$. cinerea, general ROS quenching would not be advantageous for a necrotrophic pathogen. We propose instead that B. cinerea secretes mannitol primarily as a protection against the direct antimicrobial effects of the oxidative burst. Because the oxidative burst is transient, high localized concentrations of mannitol around the fungal structures and spores themselves could be sufficient to quench this ROS and protect the fungus during this period. Conversely, if this protective mannitol is metabolized prior to or during the oxidative burst, then the plant's antimicrobial ROS could act to kill the pathogenprior to induction of visible HR. This is consistent with the observation that leaves of high MTD expressers (e.g. plant Tom-2, Figure 1) often showed virtually no necrosis in detached leaf assays.

Interestingly, while the vast majority of transgenic plants in this study had decreased B. cinerea lesion formation in detached leaf assays that were proportional to levels of MTD expression, there were two exceptions. Transgenic plant 44 had by far the highest MTD levels but had lesion size and development nearly identical to 
WT (Figure 2). Although not as striking, plant 41, which had the second highest level of MTD accumulation, had only a moderate reduction in lesion development. Although both plants appeared phenotypically normal, several reported possibilities might explain the observed response. These range from possible defects in the MTD protein itself that effect enzyme activity or localization/transport, to previously proposed detrimental effects of extreme protein overexpression. These effects have been reported to include the combined effects of diverting resources from critical cellular processes, as well as overtaxing cellular mechanisms such as translation (reviewed in [34] [35]).

Although expression of MTD above a certain level can be apparently detrimental, the preponderance of our data support the hypothesis that mannitol is an important pathogenicity factor, and that the pathogen-induced expression and secretion of MTD is a common defense response in plants. In many cases, however, plants that make and secrete MTD are still susceptible to mannitol-secreting pathogens such as B. cinerea, suggesting that MTD is induced both too late and in insufficient quantities to effectively counter the infection process. Thus, the constitutive overexpression of MTD used here provides a more effective resistance strategy than induced expression of endogenous MTD. Although not the first demonstration of the efficacy of MTD overexpression in engineering pathogen resistance, our results confirm and extend the idea that MTD overexpression can be an effective global strategy for engineering resistance against the apparently extensive group of fungal pathogens that make and secrete mannitol as part of the infection process.

\section{Conclusion}

In addition to providing specific validation of the ability of MTD overexpression to confer resistance to Botrytis Grey Mold in a greenhouse tomato, these results support our previous hypothesis that the secretion of MTD during infection might be a general response to mannitol secreting, necrotrophic fungal pathogensby a wide variety of plants. Thus, MTD overexpression might not only have broad application for engineering resistance to many fungal pathogens for which specific resistance has not been identified, but that selection of breeding lines with high levels of endogenous MTD expression could provide a useful and efficient means of screening populations for potential resistance to fungal pathogens.

\section{Acknowledgements}

This work is funded by a specialty crops block grant from the NCDA to JW, GA and DP. We thank Dr. Heather Olson and Dr. Mike Benson, Department of Plant Pathology, NCSU for B. cinerea cultures, and Dr. Emily Griffith and Mr. Andrew Wilcox, Department of Statistics, NCSU for help with statistical analyses. Tomato transformation is performed at the NCSU Plant Transformation Laboratory.

\section{References}

[1] van Kan, J.(2005) Infection Strategies of Botrytis cinerea. Acta Horticulturae, 669, 77-89.

[2] Nakajima, M. and Akutsu, K. (2014) Virulence Factors of Botrytis cinerea. Journal of General Plant Pathology, 80, 1523. http://dx.doi.org/10.1007/s10327-013-0492-0

[3] Levine, A., Tenhaken, R., Dixon, R. and Lamb, C. (1994) $\mathrm{H}_{2} \mathrm{O}_{2}$ from the Oxidative Burst Orchestrates the Plant Hypersensitive Disease Resistance Response. Cell, 79, 583-593. http://dx.doi.org/10.1016/0092-8674(94)90544-4

[4] Torres, M. (2010) ROS in Biotic Interactions. Physiologia Plantarum, 178, 414-429. http://dx.doi.org/10.1111/j.1399-3054.2009.01326.x

[5] Gorvin, M. and Levine, A. (2000) The Hypersensitive Response Facilitates Plant Infection by the Necrotrophic Pathogen Botrytis cinerea. Current Biology, 10, 751-757. http://dx.doi.org/10.1016/S0960-9822(00)00560-1

[6] Yu, I., Parker, J. and Bent, A. (1998) Gene for Gene Resistance without the Hypersensitive Response in Arabidopsis dnd1 Mutant. Proceedings of the National Academy of Sciences of the United States of America, 95, 7819-7824. http://dx.doi.org/10.1073/pnas.95.13.7819

[7] Rolke, Y., Liu, S., Quidde, T., Williamson, B., Schouten, A., Weltring, K., Siewers, V., Tenberge, K., Tudzynski, B. and Tudzynski, P. (2004) Functional Analysis of $\mathrm{H}_{2} \mathrm{O}_{2}$-Generating Systems in Botrytis cinerea: The Major Cu-Zn-Superoxide Dismutase (BcSOD1) Contributes to Virulence on French Bean, Whereas a Glucose Oxidase (BcGOD1) Is Dispensable. Molecular Plant Pathology, 5, 17-27. http://dx.doi.org/10.1111/j.1364-3703.2004.00201.x

[8] Segmüller, N., Kokkelink, L., Giesbert, S., Odinius, D., Kan, J. and Tudzynski, P. (2008) NADPH Oxidases Are involved in Differentiation and Pathogenicity in Botrytis cinerea. Molecular Plant-Microbe Interactions, 21, 808-819. 
http://dx.doi.org/10.1094/MPMI-21-6-0808

[9] Williamson, J., Desai, A., Krasnyanski, S., Ding, F., Guo, W., Nguyen, T., Olson, H., Dole, J. and Allen, G. (2013) Overexpression of Mannitol Dehydrogenase in Zonal Geranium Confers Increased Resistance to the Mannitol Secreting Fungal Pathogen Botrytis cinerea. Plant Cell, Tissue and Organ Culture (PCTOC), 115, 367-375.

[10] Jennings, D., Daub, M., Pharr, D. and Williamson, J. (2002) Constitutive Expression of a Celery Mannitol Dehydrogenase in Tobacco Enhances Resistance to the Mannitol-Secreting Fungal Pathogen Alternaria alternata. The Plant Journal, 32, 41-49. http://dx.doi.org/10.1046/j.1365-313X.2001.01399.x

[11] Voegele, R., Hahn, M. and Mendgen, K. (2005) Possible Roles for Mannitol and Mannitol Dehydrogenase in the Biotrophic Plant Pathogen Uromyces fabae. Plant Physiology, 137, 190-198. http://dx.doi.org/10.1104/pp.104.051839

[12] Joosten, M., Hendrickx, L. and de Wit, P. (1990) Carbohydrate Composition of Apoplastic Fluids Isolated from Tomato Leaves Inoculated with Virulent or Avirulent Races of Cladosporium fulvum (Syn. Fulvia fulva). Netherlands Journal of Plant Pathology, 96, 103-112. http://dx.doi.org/10.1007/BF02005134

[13] Smirnoff, N. and Cumbes, Q.J. (1989) Hydroxyl Radical Scavenging Activity of Compatible Solutes. Phytochemistry, 28, 1057-1060. http://dx.doi.org/10.1016/0031-9422(89)80182-7

[14] Chaturvedi, V., Bartiss, A. and Wong, B. (1997) Expression of Bacterial $m t l D$ in Saccharomyces cerevisiae Results in Mannitol Synthesis and Protects a Glycerol-Defective Mutant from High-Salt and Oxidative Stress. Journal of Bacteriology, 179, 157-162.

[15] Vélëz, H., Glassbrook, N. and Daub, M. (2008) Mannitol Biosynthesis Is Required for Plant Pathogenicity by Alternaria alternata. FEMS Microbiology Letters, 285, 122-129. http://dx.doi.org/10.1111/j.1574-6968.2008.01224.x

[16] Stoop, J., Williamson, J. and Pharr, D. (1996) Mannitol Metabolism in Plants: A Method for Coping with Stress. Trends in Plant Science, 1, 139-144. http://dx.doi.org/10.1016/S1360-1385(96)80048-3

[17] Stoop, J. and Pharr, D. (1992) Partial Purification and Characterization of Mannitol: Mannose 1-Oxidoreductase from Celeriac (Apium graveolens var. rapaceum) Roots. Archives of Biochemistry and Biophysics, 298, 612-619. http://dx.doi.org/10.1016/0003-9861(92)90456-7

[18] Williamson, J., Massel, M., Conkling, M. and Pharr, D. (1995) Sequence Analysis of a Mannitol Dehydrogenase cDNA from Plants Reveals a Function for the Pathogenesis-Related Protein ELI3. Proceedings of the National Academy of Sciences of the United States of America, 92, 7148-7152. http://dx.doi.org/10.1073/pnas.92.16.7148

[19] Jennings, D., Ehrenshaft, M., Pharr, M. and Williamson, J. (1998) Roles for Mannitol and Mannitol Dehydrogenase in Active Oxygen-Mediated Plant Defense. Proceedings of the National Academy of Sciences of the United States of America, 95, 15129-15133. http://dx.doi.org/10.1073/pnas.95.25.15129

[20] Lauter, F. (1996) Root-Specific Expression of the LeRse-1 Gene in Tomato Is Induced by Exposure of the Shoot to Light. Molecular Genetics and Genomics, 252, 751-754.

[21] Kiedrowski, S., Kawalleck, P., Hahlbrock, K., Somssich, I. and Dangl, J. (1992) Rapid Activation of a Novel Plant Defense Gene Is Strictly Dependent on the Arabidopsis RPM1 Disease Resistance Locus. The EMBO Journal, 11, 46774684.

[22] Cheng, F.-Y., Zamski, E., Guo, W.-W., Pharr, D. and Williamson, J. (2009) Salicylic Acid Stimulates Secretion of the Normally Symplastic Enzyme Mannitol Dehydrogenase (MTD): A Possible Defense against Mannitol-Secreting Fungal Pathogens. Planta, 230, 1093-1103. http://dx.doi.org/10.1007/s00425-009-1006-3

[23] Blackburn, K., Cheng, F., Williamson, J. and Goshe, M. (2010) Data-Independent Liquid Chromatography/Mass Spectrometry (LC/MSE) Detection and Quantification of the Secreted Apium graveolens Pathogen Defense Protein Mannitol Dehydrogenase. Rapid Communications in Mass Spectrometry, 24, 1009-1016. http://dx.doi.org/10.1002/rcm.4476

[24] Murashige, T. and Skoog, F. (1962) A Revised Medium for Rapid Growth and Bio Assays with Tobacco Tissue Cultures. Physiologia Plantarum, 15, 473-497. http://dx.doi.org/10.1111/j.1399-3054.1962.tb08052.x

[25] Gamborg, O., Miller, R. and Ojima, K. (1968) Nutrient Requirements of Suspension Cultures of Soybean Root Cells. Experimental Cell Research, 50, 151-158. http://dx.doi.org/10.1016/0014-4827(68)90403-5

[26] Bradford, M. (1976) A Rapid and Sensitive Method for the Quantitation of Microgram Quantities of Protein Utilizing the Principle of Protein-Dye Binding. Analytical Biochemistry, 72, 248-252. http://dx.doi.org/10.1016/0003-2697(76)90527-3

[27] Laemmli, U. (1970) Cleavage of Structural Proteins during the Assembly of the Head of Bacteriophage T4. Nature, 227, 680-685. http://dx.doi.org/10.1038/227680a0

[28] Stoop, J., Williamson, J., Conkling, M. and Pharr, D. (1995) Purification of NAD-Dependent Mannitol Dehydrogenase from Celery Suspension Cultures. Plant Physiology, 108, 1219-1225. http://dx.doi.org/10.1104/pp.108.3.1219

[29] Spurr Jr., H. (1973) An Efficient Method for Producing and Studying Tobacco Brown-Spot Disease in the Laboratory. Tobacco Science, 17, 145-148. 
[30] Floryszak-Wieczorek, J., Arasimowicz, M., Milczarek, G., Jelen, H. and Jackowiak, H. (2007) Only an Early Nitric Oxide Burst and the Following Wave of Secondary Nitric Oxide Generation Enhanced Effective Defense Responses of Pelargonium to a Necrotrophic Pathogen. New Phytologist, 175, 718-730. http://dx.doi.org/10.1111/j.1469-8137.2007.02142.x

[31] Barna, B. and Györgyi, B. (1992) Resistance of Young versus Old Tobacco Leaves to Necrotrophs, Fusaric Acid, Cell Wall Degrading Enzymes and Autolysis of Membrane Lipids. Physiological and Molecular Plant Pathology, 40, 247257. http://dx.doi.org/10.1016/0885-5765(92)90075-7

[32] Dulermo, T., Rascle, C., Billon-Grand, G., Gout, E., Bligny, R. and Cotton, P. (2010) Novel Insights into Mannitol Metabolism in the Fungal Plant Pathogen Botrytis cinerea. Biochemical Journal, 427, 323-332. http://dx.doi.org/10.1042/BJ20091813

[33] Ceccaroli, P., Saltarelli, R., Guescini, M., Polidori, E., Buffalini, M., Menotta, M., Pierleoni, R., Barbieri, E. and Stocchi, V. (2007) Identification and Characterization of the Tuber borchii D-Mannitol Dehydrogenase Which Defines a New Subfamily within the Polyol-Specific Medium Chain Dehydrogenases. Fungal Genetics and Biology, 44, 965-978. http://dx.doi.org/10.1016/j.fgb.2007.01.002

[34] Glick, B. (1995) Metabolic Load and Heterologous Gene Expression. Biotechnology Advances, 13, $247-261$. http://dx.doi.org/10.1016/0734-9750(95)00004-A

[35] Howell, S. (2013) Endoplasmic Reticulum Stress Responses in Plants. Annual Review of Plant Biology, 64, 477-499. http://dx.doi.org/10.1146/annurev-arplant-050312-120053 